\title{
FE-analysis of stresses in a Francis turbine runner at Derbendikhan power station
}

\author{
R. A. Saeed ${ }^{1}$, A. N. Galybin ${ }^{2,3}$ \& V. Popov ${ }^{2}$ \\ ${ }^{1}$ University of Sulaimani, Iraq \\ ${ }^{2}$ Wessex Institute of Technology, UK \\ ${ }^{3}$ Institute of Physics of the Earth, Russia
}

\begin{abstract}
This paper investigates the hydraulic induced stresses in a complete Francis runner at Derbendikhan power station by using Finite Element Analysis (FEA). Computations of water pressure were carried out with appropriate boundary and specific turbine loadings conditions obtained from CFD analysis. FE-analysis was carried out to compute stress distribution in the runner blades. This paper discusses combination of fluid and structure model, furthermore, this paper also discusses the maximum stress location on the blade of the runner. The results show that the maximum stresses caused by the hydraulic forces are situated at the transition between the blades and the crown/band on the trailing edge. This explains why this region has been identified as a critical area for fatigue crack initiation in the Francis turbine runner.
\end{abstract}

Keywords: Francis turbine runner, fatigue cracks, FEM stress analysis.

\section{Introduction}

In many large hydraulic turbine runners, fatigue cracks have occurred after they were put into production. Developments of fatigue cracks in the runner are mainly due to static and dynamic stresses in the runner. Stress analysis of hydraulic turbine runner can only be performed by numerical methods due to the complexity of these structures. Finite Element modelling has been used by many researchers, e.g. by Angehrn et al. [1] to calculate stresses in a blade of the model turbine for different distributions of hydraulic loadings and by Nava et al. [2] to find hydraulic induced stresses in Francis runner. Comparison finite element analysis with strain gauge measurements in an operating Francis 
turbine was presented by [1, 3-6], the calculated results obtained by the finite element method were in satisfactory agreement with the experimental results.

In this paper, a Finite Element Method (FEM) simulation of the complete Francis turbine runner was performed to calculate the stresses in the Francis turbine runner at low head operating condition. The loads were obtained from the CFD analysis for specific boundary conditions are incorporated into Finite Element model to calculate stress distributions in the runner.

\section{Computational fluid dynamics (CFD) simulations}

Computational Fluid Dynamics (CFD) simulation of the Francis turbine runner was performed in our previous study [7] to obtain pressure distribution and flow velocity through the turbine runner for specific operating condition. Inlet boundary conditions at given performance point of the hydraulic turbine runner are derived from an operation condition which include discharge Q $\left(82 \mathrm{~m}^{3} / \mathrm{s}\right)$, where the Guide Vanes opening is $80 \%$. Outlet boundary condition is defined to an opening with an average relative pressure to atmospheric pressure.

For the computational domain, the Spiral Casing, Stay Vane, Guide Vane, Runner and upper part of the Draft tube was considered. According to the provided specifications from Derbendikhan hydropower station a threedimensional geometrical model has been created, as shown in Figures 1 and 2. The geometry of the fluid domain has been created on AutoCAD software and inserted into ANSYS software.

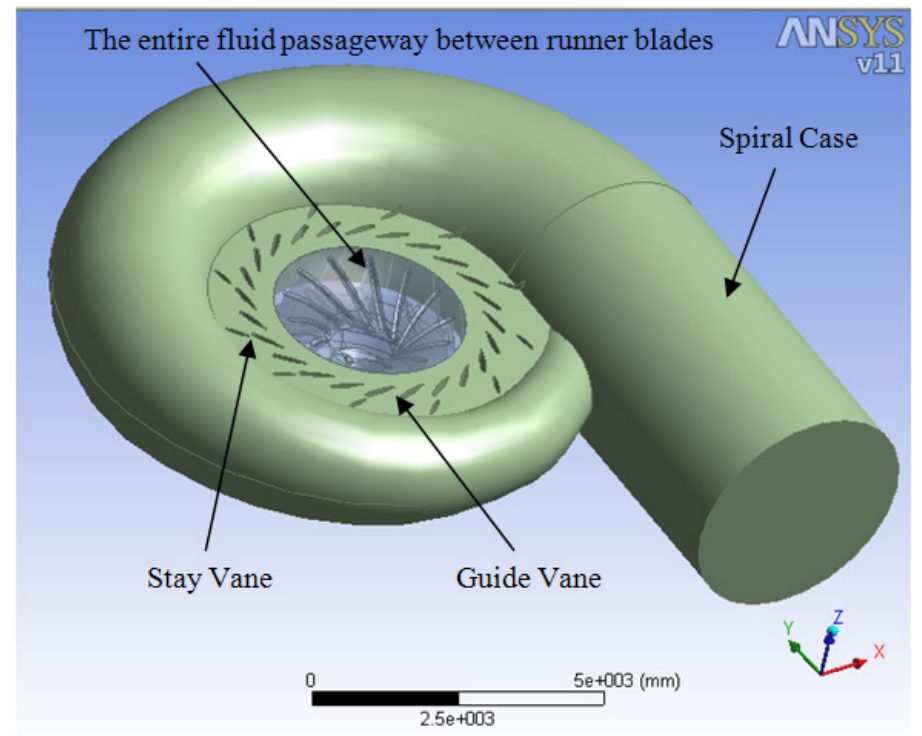

Figure 1: $\quad$ Geometrical model used in the simulation [7]. 


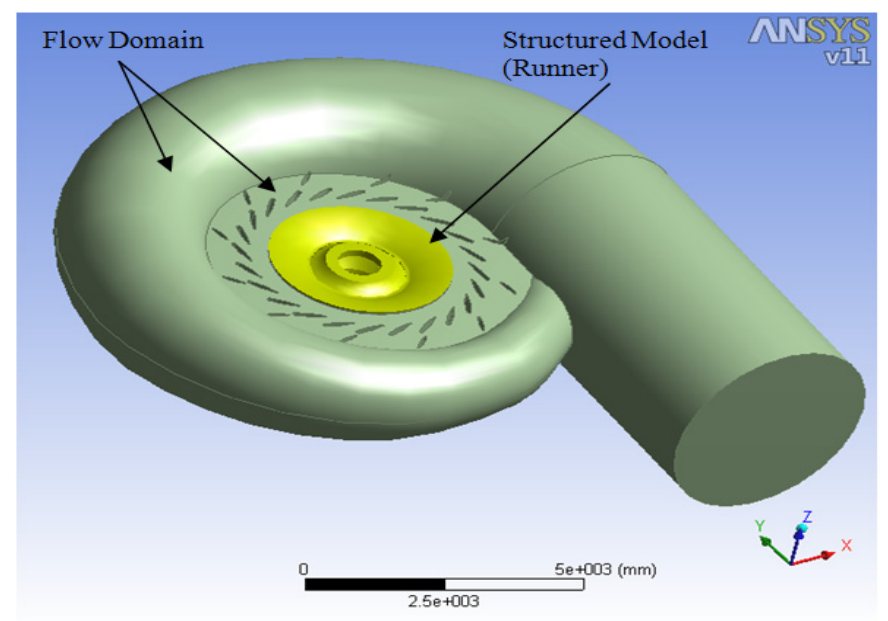

Figure 2: $\quad$ Flow domain and structured model [7].

Three-dimensional analyses of fluid flow have been carried out. The variation of pressure distributions and tangential velocity in the computational domain obtained from the CFD analysis for specific operating conditions are computed.

As illustrated in Figure 3, the flow is coming out from the spiral inlet to the upper part of the draft tube at the outlet. The flow is streamlined up to the runner outlet and recirculation is established in the draft tube. The symmetrical inflow to the runner in circumferential direction can be observed, which results in symmetrical distribution pressure from the uniform flow distribution of the runner inlet. The pressure profiles are used further on to study stresses distribution in the turbine runner.

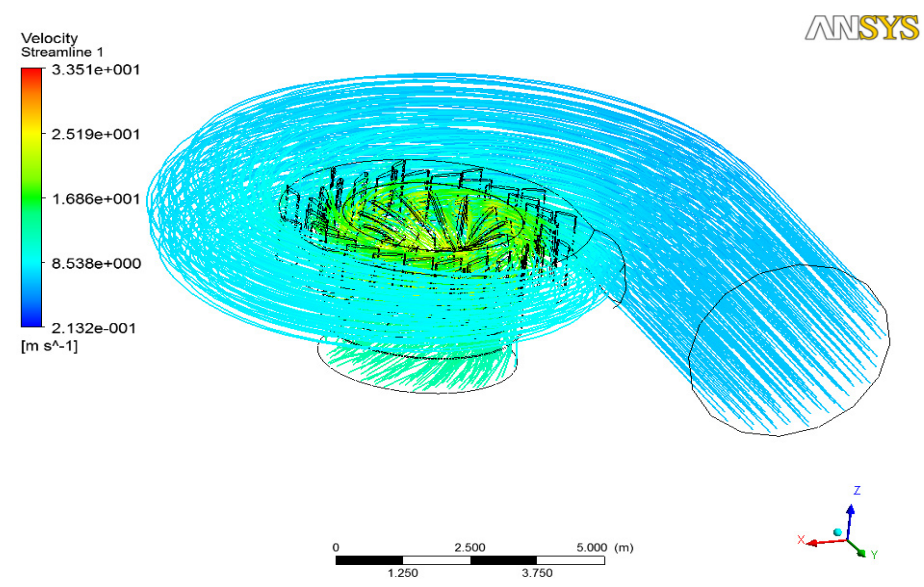

Figure 3: Tangential velocity $\left[\mathrm{ms}^{-1}\right]$ of the computational domain [7]. 


\section{Stress analyses in Francis turbine runner}

Stress analysis of the runner is an important issue that provides structural integrity of the turbine. In this study the analysis has been performed by using ANSYS software that an effective tool for modelling stresses in Francis turbine runners, e.g. [2, 8]. A number of tests have been performed in order to establish the degree of accuracy to be expected before carrying out the runner analysis. Three-dimensional geometric model were built for Francis turbine runner in order to calculate the stresses in the structural field, as explain in the following section.

\subsection{Geometry model}

The geometric model used in this study is shown in Figure 4, which is consists of three distinct parts: crown, blades and band. The model has been created according to the dimensions taken from the runner in field. The geometry of crown and band follow the drawings, however the surfaces of the blade were modelled by transferred points from the prototype. Since stress concentrations often occur between the blades and crown/band, the fillet of the runner blade with the runner crown and the runner band were modeled accurately. The geometry of the turbine runner has been created on AutoCAD software and inserted into ANSYS software.
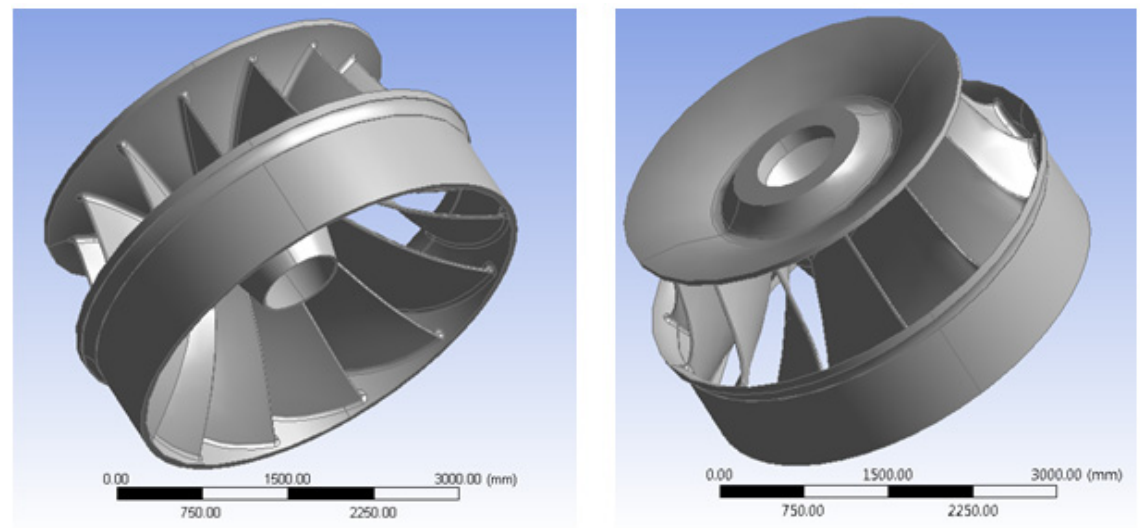

Figure 4: $\quad$ Model of the runner.

\subsection{Discretization process}

A discretized domain was generated for the whole turbine runner. The geometric model used $3 D$ tetrahedral elements. The number of elements was limited by hardware capabilities and reasonable amounts of time needed to solve the problem. It is important to mention that during the discretization process, a comparison of the convergence has been carried out with respect to mesh density 
in order to evaluate the further solution and time of computations. The whole domain was modelled with more than 270676 elements and 400930 nodes. The complete $3 D$ finite element model of the runner is shown in Figure 5.
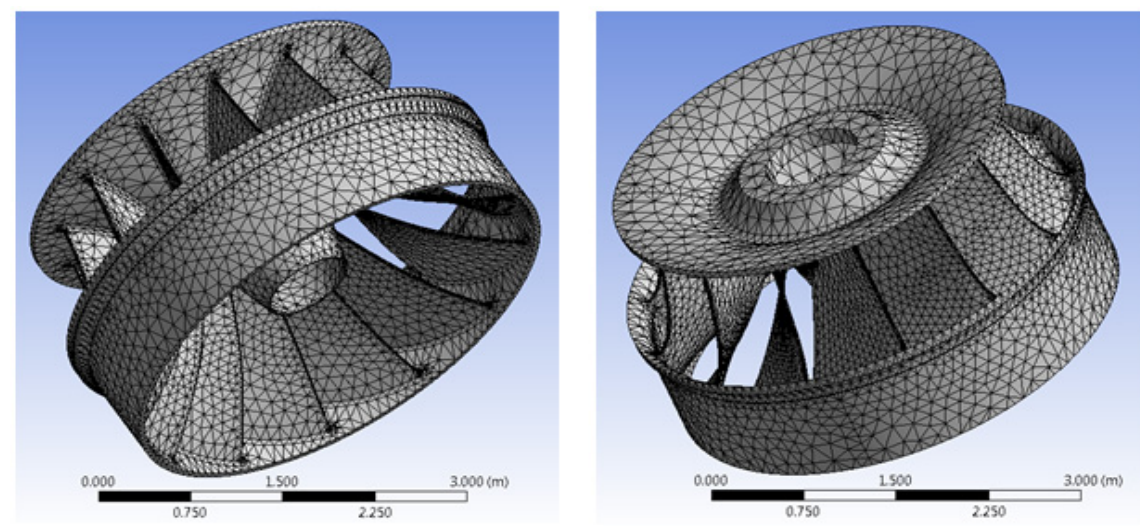

Figure 5: $\quad$ Finite element mesh of the runner model used in the analysis.

To improve the accuracy in calculation, smaller elements were used into places, where higher gradients of stresses are expected. Normally, at the tailing edge near the crown of the blades more elements were generated compared to the other parts of the model. Figure 6 illustrate the computational grid in the tailing edge of the blades near the crown.

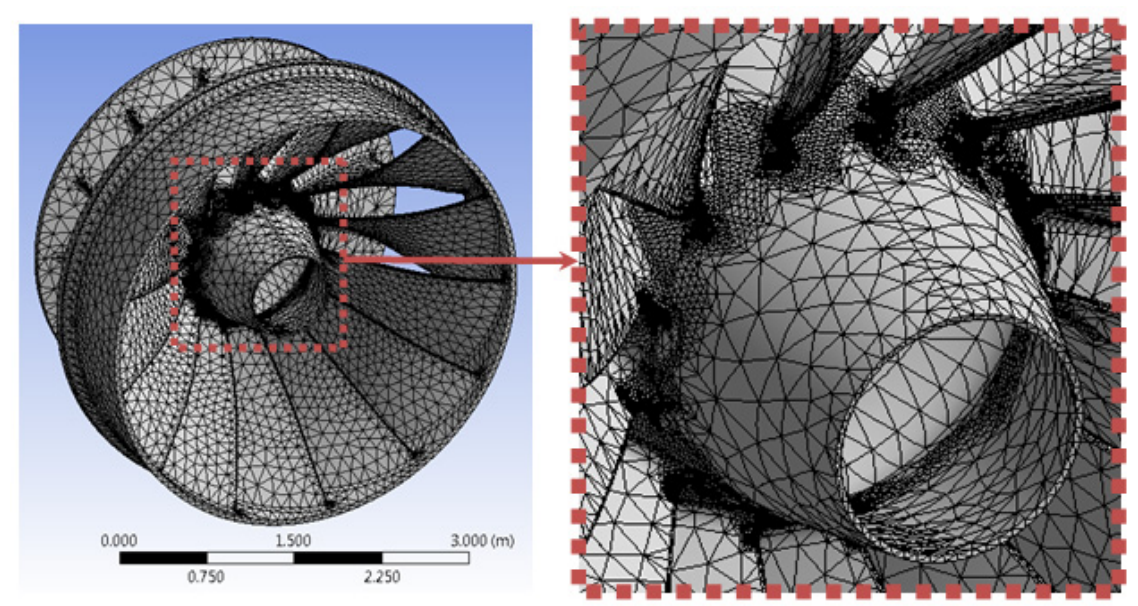

Figure 6: $\quad$ Surface grid refinement in the joint between blade and crown. 


\subsection{Loading condition}

Under normal operating conditions, a Francis turbine runner is subjected to two sorts of static loads, namely, the centrifugal force due to the rotational speed and the load due to the water pressure. In the Francis turbines, the stresses due to centrifugal force during normal operation is much smaller than the stresses due to water pressure $[2,8]$, therefore centrifugal forces are neglected further on. The loading due to water pressure on the blades are considered and the pressure loading has been derived from CFD computations. Computational fluid dynamics $(C F D)$ simulations were run at specific operating condition to analyze the pressure distribution on the runner surface.

In order to calculate the stresses in the Francis turbine runner, a $3 D C F D$ simulation combined with $3 D$ FEM simulation. The combination of CFD model and FEM model is illustrated in schematic shown in Figure 7.

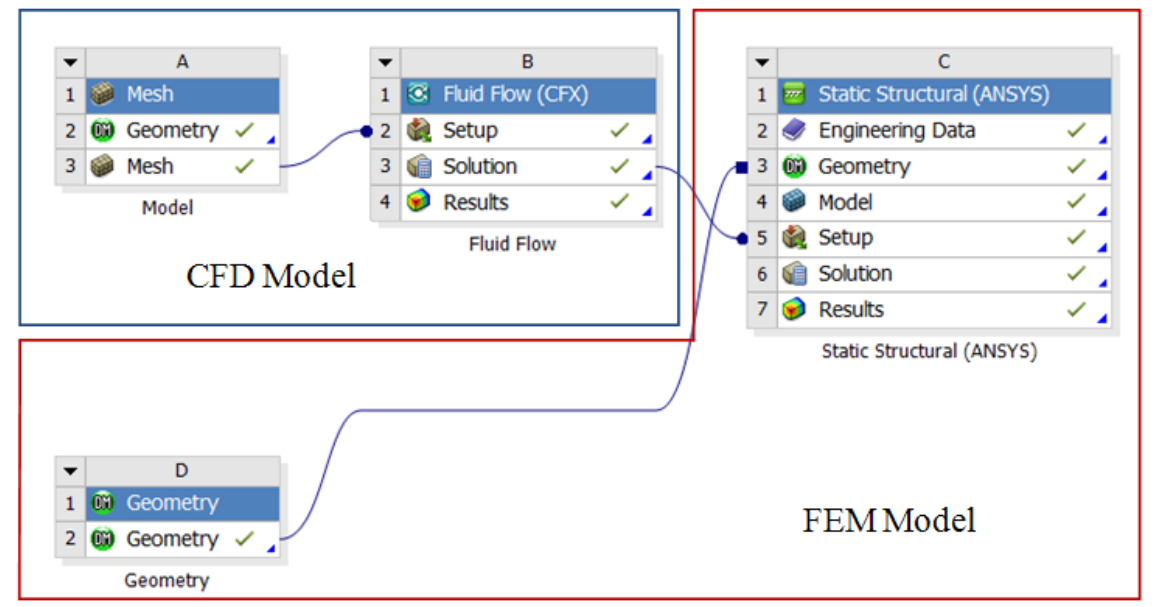

Figure 7: $\quad$ Project schematic.

\subsection{Boundary conditions}

To ensure that the model acts the same way as the prototype, the constraints and appropriate boundary conditions were applied for the runner. Displacement boundary conditions are needed to fixed between the runner crown and the main shaft, the runner will not be allowed to rotate at the support therefore the displacement vectors are zero $\left(U_{x}=U_{y}=U_{z}=0\right)$. The pressure distribution on the fluid structure interface come from the water flow in the runner, as demonstrate in Figure 8, it is computed from CFD analysis for specific operating conditions. Therefore $\sigma_{n}=P_{C F D}$, where $\sigma_{n}$ and $P$ are water pressure and normal stress, shear stresses components are set to zero. 

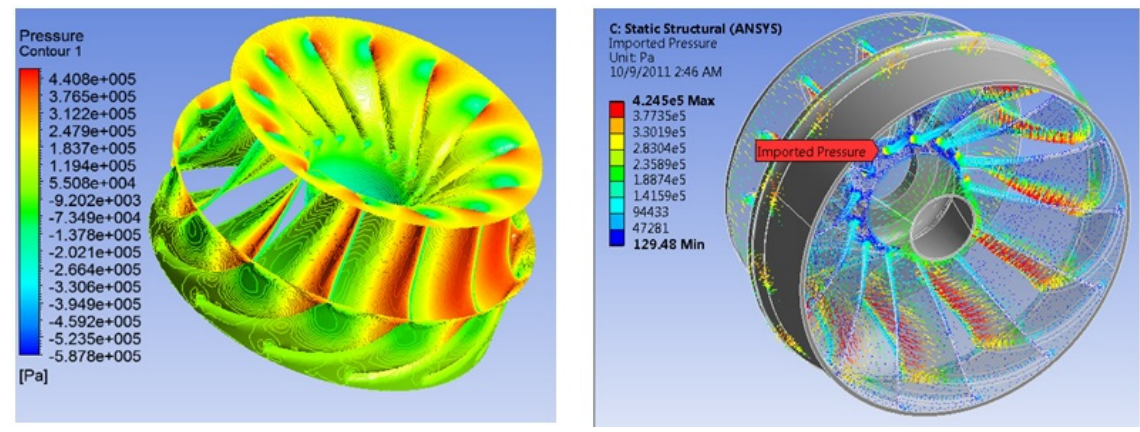

Figure 8: $\quad$ Pressure distribution on the runner's surface.

\subsection{Analysis result and discussion}

The $3 D$ FEM for the complete structure of a Francis runner has been performed by ANSYS to evaluate stress distribution in the Francis turbine's runner for specific operating condition. The stress distributions are shown in Figure 9, as evident from the figure, the maximum stresses are concentrate at the transition between the leading edge and the runner band as well as on the trailing edge close to the runner crown. Similar stress distributions are found for other Francis turbine runners $[5,6,8,9]$. The blades are deformed by water pressure in the circumferential direction and toward the turbine axis. The highest stresses on the outlet edge are mainly due to radial tension.

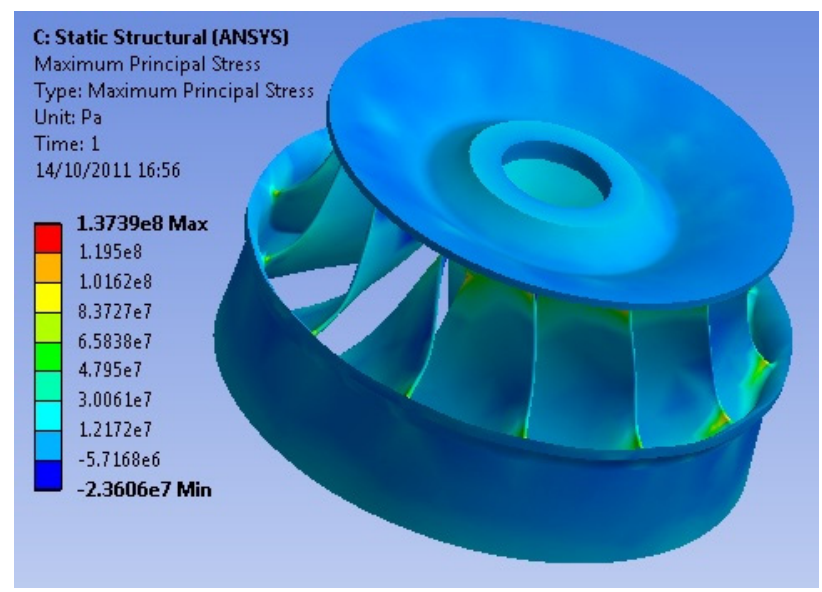

Figure 9: $\quad$ Instantaneous stress distribution on the Francis turbine runner.

The results of the stress distributions in the pressure side of the blade are shown in Figure 10. The rapid increase of stresses along the tailing edge toward the crown was observed. The highest stress values in the runner were found near the trailing edge of the blade, which causes tension in pressure side. The 
maximum principle stress is $137.39 \mathrm{~N} / \mathrm{mm}^{2}$ at the blade outlet edge near the transition from blade to crown, the principle stress gradually decreased as the distance from the trailing edge increased. The stresses in the middle of the blade are small; they do not exceed $10 \mathrm{~N} / \mathrm{mm}^{2}$.
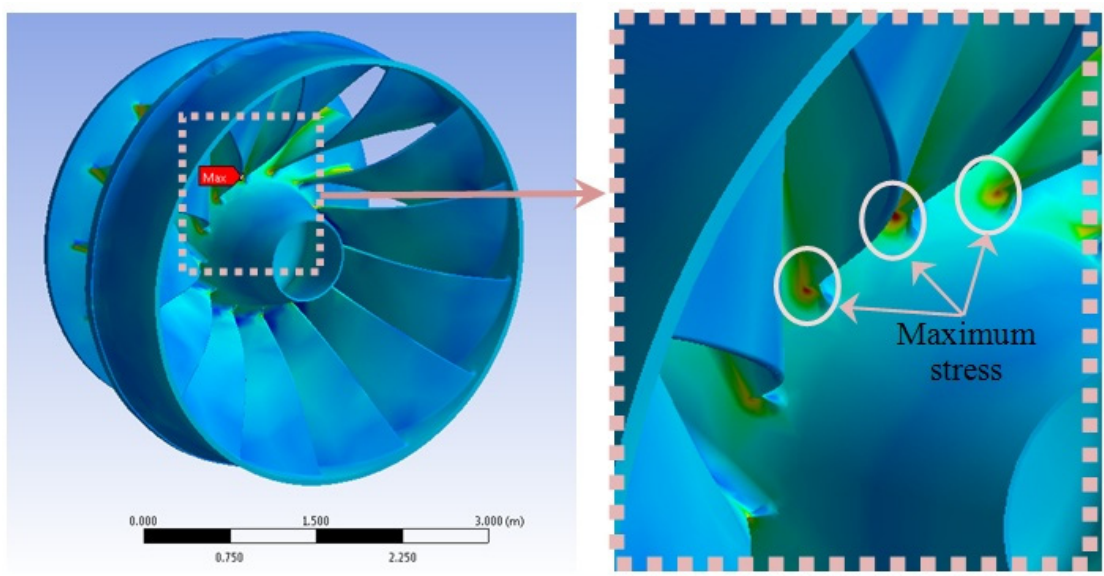

Figure 10: Stress distribution on the pressure sides of the blade for specific cases obtained by the FEM calculations.

During periodic inspections for Unit 2 in Derbendikhan power station, many fatigue cracks are observed in the runner blades. Example of these cracks is shown in Figure 11. Generally, in the Francis turbine runner the region near the trailing edge of the runner blade towards the crown has been identified as the one with high stresses and it's a critical area for fatigue crack initiation. From the numerical analysis we conclude that the maximum computed stresses are located at the transition between blade and crown on the outlet side (see Figure 10) which is the location of cracks as demonstrated in Figure 11.
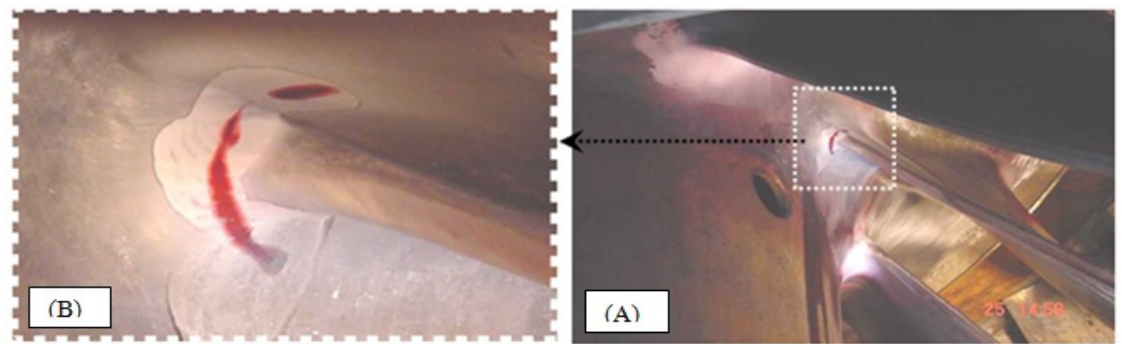

Figure 11: Cracks in the turbine runner of unit 2 in Derbendikhan power station. 


\section{Conclusions}

Stress analysis of the Francis turbine runner model has been performed. It has been found that the maximum stresses due to the water pressure are located at the trailing edge of the runner blade towards the transition between the blade and the crown. This explains why this region has been identified as a critical area for fatigue crack initiation in the Francis turbine runner. The results show good agreement with the previous studies.

\section{References}

[1] Angehrn, R., Holler, K. and Barp, B., A comparison of theoretical stress calculation with experimental verified stresses on Francis turbine runners of high specific speed, Escher Wyss News, Vol. 50, No. 1, pp. 25-28, 1977.

[2] Nava, J. M. F., Gómez, O. D., Hernández J. A. R. L., Flow induced stresses in a Francis runner using ANSYS, International ANSYS Conference Proceedings, 2006.

[3] Farhat, M., S. Natal, Avellan, F., Paquet, F., Lowys, Py., Couston, M., Onboard measurements of pressure and strain fluctuations in a model of low head Francis turbine part 2 : Measurements and preliminary analysis results, Proceedings of the $21^{\text {st }}$ IAHR Symposium on Hydraulic Machinery and Systems, Lausanne, pp. 873-880, 2002.

[4] Ikeda, K., Inagaki, M., Niikura, K., Oshima, K., 700-m 400-MW class Ultrahigh-head Pump Turbine, Hitachi Review Vol. 49, No. 2, pp. 81-87, 2000.

[5] Bjorndal, H., Moltubakk, T. and Aunemo, H., Flow induced stresses in a medium head Francis runner - strain gauge measurements in an operating plant and comparison with finite eElement analysis, $10^{\text {th }}$ International Meeting of the IAHR Work Group on the Behaviour of Hydraulic Machinery Under Steady Oscillatory Conditions, Trondheim, Norway, 2001.

[6] Ivanchenko, I. P., Smelkov, L. L., Pupko, T. E., Timashkov, A. Ya., Vapnik, B. K., Stress-strain state of Francis turbine blades, Power Technology and Engineering, Springer, New York, pp. 755-762, 1982.

[7] Saeed, R. A., Galybin, A. N., Popov, V., Complete Francis Turbine Flow Simulation at Derbendikhan Power Station. Advances in Fluid Mechanics, Proceedings of the Advances in Fluid Mechanics IX, Split, Croatia, WIT press, pp. 49-57, 2012.

[8] Dubas, M. and Schuch, M., Static and dynamic analysis of Francis turbine runners, Sulzer Technical Review, Switzerland, Vol. 69, No. 3, pp. 30-32, 1987.

[9] Ruofu, X., Zhengwei, W., Yongyao, L., Dynamic stresses in a Francis turbine runner based on fluid-structure interaction analysis, Tsinghua Science and Technology, Vol. 13, No. 5, pp. 587-592, 2008. 